\title{
Energy substrates that fuel fast neuronal network oscillations
}

\section{Lukas V. Galow, Justus Schneider, Andrea Lewen, Thuy-Truc Ta, Ismini E. Papageorgiou ${ }^{\dagger}$ and Oliver Kann*}

Institute of Physiology and Pathophysiology and Interdisciplinary Center for Neurosciences (IZN), University of Heidelberg, Heidelberg, Germany

\section{Edited by:}

Avital Schurr, University of

Louisville, USA

Reviewed by:

Neil Sims, Flinders University, Australia

David Mazzocchi-Jones, Keele University, UK

\section{*Correspondence:}

Oliver Kann, Institute of Physiology and Pathophysiology, University of Heidelberg, Im Neuenheimer Feld 326, D-69120 Heidelberg, Germany e-mail:oliver.kann@

physiologie.uni-heidelberg.de

${ }^{\dagger}$ Present address:

Ismini E. Papageorgiou, Institute for Diagnostic and Interventional Neuroradiology, University of Göttingen, Göttingen, Germany
Fast neuronal network oscillations in the gamma-frequency band $(30-100 \mathrm{~Hz})$ provide a fundamental mechanism of complex neuronal information processing in the hippocampus and neocortex of mammals. Gamma oscillations have been implicated in higher brain functions such as sensory perception, motor activity, and memory formation. The oscillations emerge from precise synapse interactions between excitatory principal neurons such as pyramidal cells and inhibitory GABAergic interneurons, and they are associated with high energy expenditure. However, both energy substrates and metabolic pathways that are capable to power cortical gamma oscillations have been less defined. Here, we investigated the energy sources fueling persistent gamma oscillations in the CA3 subfield of organotypic hippocampal slice cultures of the rat. This preparation permits superior oxygen supply as well as fast application of glucose, glycolytic metabolites or drugs such as glycogen phosphorylase inhibitor during extracellular recordings of the local field potential. Our findings are: (i) gamma oscillations persist in the presence of glucose $(10 \mathrm{mmol} / \mathrm{L})$ for greater than $60 \mathrm{~min}$ in slice cultures while (ii) lowering glucose levels $(2.5 \mathrm{mmol} / \mathrm{L})$ significantly reduces the amplitude of the oscillation. (iii) Gamma oscillations are absent at low concentration of lactate $(2 \mathrm{mmol} / \mathrm{L}$ ). (iv) Gamma oscillations persist at high concentration $(20 \mathrm{mmol} / \mathrm{L})$ of either lactate or pyruvate, albeit showing significant reductions in the amplitude. (v) The breakdown of glycogen significantly delays the decay of gamma oscillations during glucose deprivation. However, when glucose is present, the turnover of glycogen is not essential to sustain gamma oscillations. Our study shows that fast neuronal network oscillations can be fueled by different energy-rich substrates, with glucose being most effective.

Keywords: brain energy metabolism, electrophysiology, glycogen phosphorylase, information processing, lactate, mitochondria, monocarboxylate transporter, synaptic transmission

\section{INTRODUCTION}

The mammalian brain is a highly oxidative organ owing to the disproportionately large fraction of oxygen consumption compared with the small fraction of the total body mass (in humans about $20 \%$ and $2 \%$, respectively) (Rolfe and Brown, 1997; Erecińska and Silver, 2001). This suggests that complex neuronal information processing is associated with high energy expenditure and requires continuous delivery of glucose from the blood (Shulman et al., 2001; Attwell et al., 2010; Kann, 2012). Glucose enters the extracellular space of the brain parenchyma based on a large concentration gradient $(5-7 \mathrm{mmol} / \mathrm{L}$ in the blood and $1-2 \mathrm{mmol} / \mathrm{L}$ in the extracellular space) via glucose transporters (GLUTs) that are located on endothelial cells of the blood-brain-barrier as well as astrocytes (Roberts, 2007; Hertz et al., 2014). For normal conditions, glucose has been considered to be the dominant exogenous energy substrate in the adult brain (Chih and Roberts, 2003; Dienel, 2012). Having a role in

Abbreviations: Ampl, amplitude; AUC, area under curve; DIV, days in vitro; Freq, peak frequency; FWHM, full width at half maximum; LFP, local field potential; PSD, peak power spectral density; Var, variance of the amplitude. different biochemical pathways, glucose metabolism has important functions related to bioenergetics, neurotransmission, and oxidation-reduction (redox) reactions in the brain parenchyma (Bak et al., 2006; Kann and Kovács, 2007; Dienel, 2012). However, neurons are able to utilize exogenous and endogenous energy substrates other than glucose in certain physiological and pathophysiological conditions (Roberts, 2007).

A prominent example is lactate that is generated during neuronal activity through glycogenolysis and/or anaerobic glycolysis (1-2 $\mathrm{mmol} / \mathrm{L}$ in the extracellular space) or may even enter the brain parenchyma from the blood (Ide et al., 2000; Roberts, 2007; Overgaard et al., 2012; Hertz et al., 2014). Lactate metabolism may also involve complex interactions between neurons and astrocytes (Suzuki et al., 2011; Pellerin and Magistretti, 2012). Many studies on lactate and neuronal activity were made in slice preparations of the hippocampus (Schurr et al., 1988, 1999; Stittsworth and Lanthorn, 1993; Galeffi et al., 2007; Schurr and Payne, 2007; Ivanov et al., 2011, 2014; Hall et al., 2012; Schurr and Gozal, 2012). In all of these studies, neuronal activation was induced by repetitive electrical stimulation, application of neurotransmitters such as glutamate, or $\mathrm{Mg}^{2+}$-free recording 
solution. Notably, either of these experimental tools evokes quite robust neuronal activation, with widely undefined activity states in the local neuronal network, or even spreading (epileptic) depolarization. Thus, detailed information about the capability of different energy substrates to fuel specific, naturally occurring network activity states such as fast neuronal network oscillations in the gamma-frequency band $(30-100 \mathrm{~Hz})$ is lacking in the literature.

Gamma oscillations $(30-100 \mathrm{~Hz})$ have been observed in many mammalian brain regions, including the hippocampus and the neocortex (Uhlhaas and Singer, 2010). Gamma oscillations reflect synchronous rhythmic fluctuations of the membrane potential in many neurons of a local neuronal network. In the hippocampus, these subthreshold fluctuations are generated by complex and precise synaptic interactions of excitatory pyramidal cells and inhibitory GABAergic interneurons (Whittington and Traub, 2003; Hájos and Paulsen, 2009; Kann, 2012). The synchronizing effect of gamma oscillations permits the coordinated activation of defined sets of neurons that carry and process information (Hájos and Paulsen, 2009; Kann et al., 2014). Therefore, gamma oscillations provide a temporal matrix for complex neuronal information processing during higher brain functions such as sensory perception, motor activity, and memory formation (Paulsen and Moser, 1998; Uhlhaas and Singer, 2010; van Vugt et al., 2010).

Notably, excitatory pyramidal cells and certain subtypes of inhibitory GABAergic interneurons might differ in the energy demands during gamma oscillations and in their capability to utilize energy substrates. Parvalbumin-positive basket cells, for example, generate action potentials at much higher frequency ("fast-spiking") compared with pyramidal cells during gamma oscillations (30-100 Hz and $1-3 \mathrm{~Hz}$, respectively). Moreover, parvalbumin-positive basket cells synchronize the activity of numerous pyramidal cells by rhythmic inhibition. As prerequisites, theses interneurons have unique electrophysiological properties that are likely associated with extraordinary high energy expenditure (Gulyás et al., 2006; Hu and Jonas, 2014; Kann et al., 2014).

The present study was designed to identify energy substrates that are capable to power gamma oscillation in vitro. Gamma oscillations can be reliably induced in hippocampal slices by bath application of low micromolar concentrations of cholinergic receptor agonists such as acetylcholine that mimics input from the septum (Fisahn et al., 1998; Kann et al., 2011). These oscillations share many features with hippocampal gamma oscillations observed in vivo (Kann, 2012). We used organotypic hippocampal slice cultures that were maintained on Biopore ${ }^{\mathrm{TM}}$ membranes in an interface recording chamber. This experimental approach permits the induction of persistent gamma oscillations, with superior oxygen supply as well as rapid exchange of energy substrates and drugs (Huchzermeyer et al., 2013).

\section{MATERIALS AND METHODS SLICE CULTURES AND RECORDING CHAMBER}

Animals were purchased from Charles-River (Sulzfeld, Germany) and housed, cared, and killed in accordance with the recommendations of the European Commission and the authorities of Baden-Württemberg (T56/11). Organotypic hippocampal slice cultures were prepared as described (Kann et al., 2003a,b, 2011). In brief, hippocampal slices $(400 \mu \mathrm{m})$ were cut with a McIlwain tissue chopper (Mickle Laboratory Engineering Company Ltd., Guildford, UK) from 7 to 9 days-old Wistar rats under sterile conditions. Slices were maintained on Biopore ${ }^{\mathrm{TM}}$ membranes (Millicell standing inserts, Merck Millipore, Schwalbach, Germany) between culture medium, which consisted of 50\% minimal essential medium, 25\% Hank's balanced salt solution (Sigma-Aldrich, Taufkirchen, Germany), 25\% horse serum (Life Technologies, Darmstadt, Germany), and 2 mM L-glutamine (Life Technologies) at pH 7.3, and humidified normal atmosphere $\left(5 \% \mathrm{CO}_{2}, 36.5^{\circ} \mathrm{C}\right)$ in an incubator (Heracell, Thermoscientific, Dreieich, Germany). Biopore ${ }^{\mathrm{TM}}$ membranes provide high viability and excellent trans-membrane oxygen transport. The culture medium $(1 \mathrm{ml})$ was replaced three times per week. Slice cultures were used after 7-21 days in vitro (DIV) (residual thickness of about $200 \mu \mathrm{m}$ ), when the tissue had recovered from the slice preparation and damaged cut surfaces were re-organized (Kann and Kovács, 2007).

For recordings, the intact Biopore ${ }^{\mathrm{TM}}$ membrane carrying slice cultures was inserted into the recording chamber. Slice cultures were maintained at the interface between recording solution and ambient gas mixture. Intact Biopore ${ }^{\mathrm{TM}}$ membrane inserts ensure constant supply of oxygen and energy substrates from the recording solution (rate $1.8 \mathrm{ml} / \mathrm{min}$ ) that flows underneath the Biopore $^{\mathrm{TM}}$ membrane; the interface condition permits constant oxygen supply from the ambient gas mixture $\left(95 \% \mathrm{O}_{2}\right.$ and $5 \%$ $\mathrm{CO}_{2}$, rate $\left.1.5 \mathrm{l} / \mathrm{min}\right)$.

\section{RECORDING SOLUTIONS AND DRUGS}

Slice cultures were constantly supplied with pre-warmed (34 \pm $1^{\circ} \mathrm{C}$ ) recording solution, i.e., artificial cerebrospinal fluid that contained: $129 \mathrm{mM} \mathrm{NaCl}, 3 \mathrm{mM} \mathrm{KCl}, 1.25 \mathrm{mM} \mathrm{NaH}_{2} \mathrm{PO}_{4}$, $1.8 \mathrm{mM} \mathrm{MgSO}_{4}, 1.6 \mathrm{mM} \mathrm{CaCl}_{2}, 26 \mathrm{mM} \mathrm{NaHCO}_{3}$, and $10 \mathrm{mM}$ glucose (Sigma-Aldrich). The $\mathrm{pH}$ was 7.3 when the recording solution was saturated with the gas mixture $\left(95 \% \mathrm{O}_{2}\right.$ and $\left.5 \% \mathrm{CO}_{2}\right)$.

Gamma oscillations were induced by bath application of low concentrations of cholinergic receptor agonist, acetylcholine $(2 \mu \mathrm{mol} / \mathrm{L})$ and acetylcholine-esterase inhibitor, physostigmine (400 nmol/L) (Huchzermeyer et al., 2013). The absence of action potentials (spiking) was induced by bath application of tetrodotoxin, which blocks fast voltage-gated $\mathrm{Na}^{+}$-channels. Acetylcholine was from Sigma-Aldrich, physostigmine was from Tocris and tetrodotoxin from Biotrend (Köln, Germany).

For further specific experiments, Na-pyruvate (SigmaAldrich), Na-L-lactate (Alfa Aesar, Karlsruhe, Germany), L-glutamine, CP-316819 (5-Chloro-N-[(1S,2R)-2-hydroxy-3(methoxymethylamino)-3-oxo-1-(phenylmethyl)propyl]-1H-indole-2-carboxamide; Tocris, R\&D Systems GmbH, WiesbadenNordenstadt, Germany) and DAB (1,4-dideoxy-1,4-imino-darabinitol; Sigma-Aldrich) were used. Stock solution of DAB was made in double distilled $\mathrm{H}_{2} \mathrm{O}$ and CP-316819 was dissolved in DMSO, with a final solvent fraction of less than $0.001 \%$ in the recording solution. 


\section{RECORDINGS OF LOCAL FIELD POTENTIALS}

The local field potential (LFP) was recorded with glass electrodes (tip diameter 3-5 $\mu \mathrm{m}$ ) that were pulled from GB150F-8P borosilicate filaments (Science Products $\mathrm{GmbH}$, Hofheim, Germany) with a PC-10 vertical micropipette puller (Narishige International Ltd., London, UK) and backfilled with recording solution. The glass electrode was positioned in stratum pyramidale of the CA3 subfield with a mechanical micromanipulator (MM 33, Märzhäuser, Wetzlar). LFPs were recorded with an EXT 10-2F amplifier in EPMS-07 housing (npi electronic $\mathrm{GmbH}$, Tamm, Germany), low-pass filtered at $3 \mathrm{kHz}$, and digitized at $10 \mathrm{kHz}$ using CED 1401 interface and Spike2 software (Cambridge Electronic Design, Cambridge, UK) for offline analysis.

\section{TOLUIDINE BLUE STAINING}

Slice cultures were fixed in paraformaldehyde (4\%, $0.1 \mathrm{M}$ phosphate buffer; Applichem, Darmstadt, Germany) and rinsed in $0.1 \mathrm{M}$ phosphate-buffered salt solution (PBS). Thereafter, slice cultures were exposed for $20 \mathrm{~min}$ to toluidine-blue working solution, which was a mixture of $5 \mathrm{ml}$ stock solution ( $1 \mathrm{~g}$ of Toluidine Blue $\mathrm{O}$ in $100 \mathrm{ml}$ of $70 \%$ ethanol; Sigma-Aldrich) and $45 \mathrm{ml}$ of $1 \% \mathrm{NaCl}$ solution ( $\mathrm{pH} 2.0-2.5)$. Thereafter, $96 \%$ ethanol $(100 \mathrm{ml}$ of $96 \%$ ethanol and 4 drops of acetic acid) was used for colordifferentiation of the staining. The differentiation step with strong acid removes unspecific staining of weak acidic structures and, thus, increases the contrast between background and stained cells. The process was stopped using 0.1 M PBS, once the differentiation was clearly visible. After brief rinsing with double distilled water, slice cultures were placed on object plates and dried overnight. The slices were then exposed to xylol (Sigma-Aldrich) for $10 \mathrm{~min}$ and embedded with Entellan Neu (Merck Millipore, Schwalbach, Germany).

\section{DATA ANALYSIS}

Offline signal analysis of gamma oscillations was performed in MatLab 11.0 (MathWorks). Data segments of $100 \mathrm{~s}$ were low-pass filtered with a digital Butterworth algorithm at $200 \mathrm{~Hz}$ corner frequency and processed with Welch's algorithm and Fast Fourier Transformation with a Hamming window size of 4096 points for calculation of the power spectral density and the power spectrum, respectively (bin size $=2.441 \mathrm{~Hz}$ ). Gamma oscillations were analyzed for various parameters, i.e., peak frequency (Freq), area under curve (AUC), full width at half maximum (FWHM), peak power spectral density (PSD), amplitude (Ampl), and variance of the amplitude (Var).

Data are presented as mean \pm SD derived from (n) slice cultures and $(\mathrm{N})$ preparations of rat pups. Statistical significance $(P<0.05)$ was determined using SigmaPlot 12.5 (Systat Software, Inc., San Jose, CA, USA). Data distribution was tested for normality with Shapiro-Wilk test. Comparisons between paired data were made with paired $t$-test or Wilcoxon signed rank test. For multiple variance comparison, One-Way ANOVA or Kruskal-Wallis One-Way ANOVA on ranks with Dunn's posthoc test was used for unpaired data and One-Way repeated measures ANOVA with Holm-Sidak post-hoc test or Friedman repeated measures ANOVA on ranks with Tukey post-hoc test was used for paired data. Figures were generated using Excel (Microsoft Corporation, Redmond, USA) and CorelDRAW (Corel Corporation, Ottawa, Ontario, Canada).

\section{RESULTS}

\section{GAMMA OSCILLATIONS IN THE PRESENCE OF GLUCOSE}

We induced gamma oscillations by bath application of acetylcholine in organotypic hippocampal slice cultures and performed extracellular recordings of the local field potential (LFP) in stratum pyramidale of the CA3 subfield (Kann et al., 2011). In standard recording solution, i.e., in the presence of glucose $(10 \mathrm{mmol} / \mathrm{L})$ and high oxygen fraction $(95 \%)$ in the ambient atmosphere (Kann and Kovács, 2007), gamma oscillations were fully established after about $15 \mathrm{~min}$ of acetylcholine application (Figures 1A-C). The oscillations were characterized by a frequency of around $40 \mathrm{~Hz}$ in the power spectrum and persisted for $60 \mathrm{~min}$ (Figure 1C) and longer (data not shown). This experiment demonstrates that the reduced composition of recording solution as well as the supply of oxygen in excess for $1 \mathrm{~h}$ does not result in evident functional disturbances in the local neuronal network of the CA3 subfield.

We next tested whether the properties of persistent gamma oscillations changed with maturation of slice cultures (Bahr et al., 1995; De Simoni et al., 2003). The characteristics of gamma oscillations in standard recording condition $(10 \mathrm{mmol} / \mathrm{L}$ glucose, $95 \%$ oxygen fraction) did not significantly change after 21 DIV (Figure 2), albeit the well-known decrease of slice thickness over time in culture (Bahr et al., 1995; Kann and Kovács, 2007). For further experiments, we used slice cultures after 7 DIV and up to 14 DIV.

Bath application of tetrodotoxin $(1 \mu \mathrm{mol} / \mathrm{l})$, which blocks fast voltage-gated $\mathrm{Na}^{+}$-channels and thus action potentials, suppressed gamma oscillations after about $5 \mathrm{~min}$ (data not shown). This experiment reveals that maintenance of slice cultures on Biopore $^{\mathrm{TM}}$ membranes in the interface recording chamber permits rapid drug application and tissue saturation.

We further addressed whether application of glucose in a concentration closer to physiological conditions $(2.5 \mathrm{mmol} / \mathrm{L})$ would affect gamma oscillations (Roberts, 2007; Schurr and Payne, 2007). In this condition, gamma oscillations were still present. However, there were significant disturbances in the characteristics of gamma oscillations such as lower amplitude (AUC, PSD, Ampl) and widening of FWHM, reflecting less numbers and less synchrony of activated synapses, respectively (Figure 3). This experiment shows that even in optimized recording condition, i.e., utilization of Biopore ${ }^{\mathrm{TM}}$ membranes and interface recording chamber, a larger concentration gradient of glucose is required to fuel gamma oscillations in vitro.

\section{LACTATE AND PYRUVATE AS ENERGY SUBSTRATES}

We next tested whether lactate in a concentration of $2 \mathrm{mmol} / \mathrm{L}$ was capable to fuel gamma oscillations, similar as reported for neuronal population responses evoked by electrical stimulation (Schurr et al., 1988). To exclude that breakdown of the glycogen reserve in slice cultures (Cater et al., 2001) affected the outcome of this experiment (see below), we first depleted the glycogen stores by glucose deprivation (recording solution with $0 \mathrm{mmol} / \mathrm{L}$ 


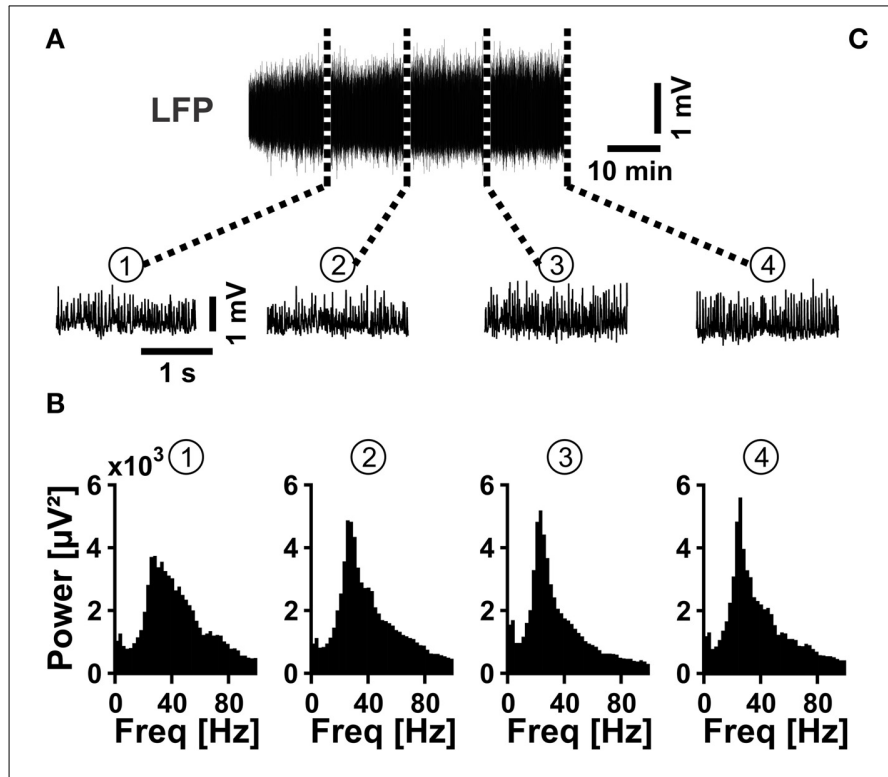

FIGURE 1 | Gamma oscillations in slice cultures. (A) Gamma oscillations were induced by bath application of acetylcholine $(2 \mu \mathrm{mol} / \mathrm{L})$ and physostigmine ( $400 \mathrm{nmol} / \mathrm{L})$ (upper sample trace), and they persisted for more than $60 \mathrm{~min}(n=13, N=6)$. Gamma oscillations are shown with a higher temporal resolution after $15 \mathrm{~min}$ (1), $30 \mathrm{~min}$ (2), $45 \mathrm{~min}$ (3), and $60 \mathrm{~min}$ (4) (lower sample traces). Local field potentials (LFP) were recorded in stratum pyramidale of the CA3 subfield in organotypic hippocampal slice
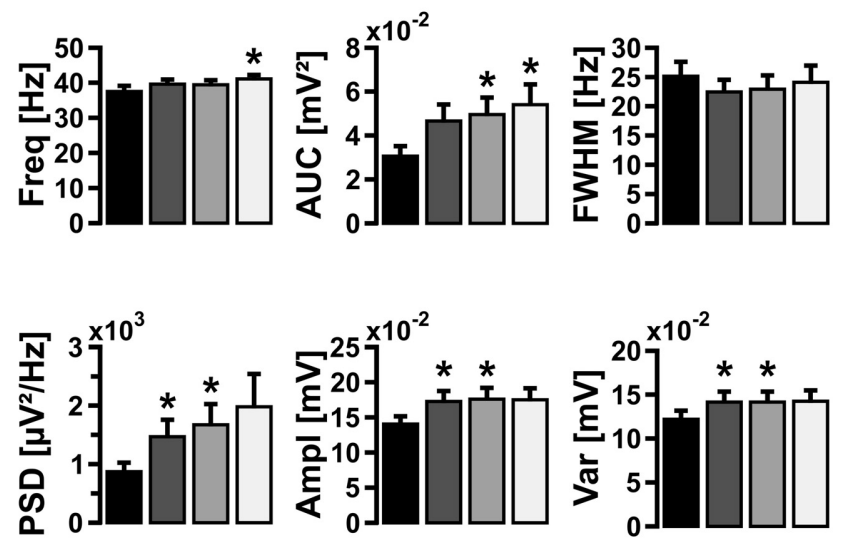

$15 \min \square 30$ min $\square 45$ min $\square 60$ min glucose) for $15 \mathrm{~min}$, which widely resulted in suppression of gamma oscillations (Figures 4A-B). Subsequent bath application of lactate $(2 \mathrm{mmol} / \mathrm{L})$ for $20 \mathrm{~min}$ did not rescue gamma oscillations. By contrast, re-application of glucose $(10 \mathrm{mmol} / \mathrm{L})$ resulted in almost full recovery of the oscillations.

Subsequently, we determined the capability of equicaloric concentrations of lactate $(20 \mathrm{mmol} / \mathrm{L})$ or pyruvate $(20 \mathrm{mmol} / \mathrm{L})$ to fuel gamma oscillations (Cater et al., 2003; Galeffi et al., 2007; Gandhi et al., 2009). Either of these substrates could indeed sustain gamma oscillations for $30 \mathrm{~min}$ (Figures 4C-F) and longer (data not shown). However, in the presence of lactate or pyruvate gamma oscillations showed significantly lower amplitudes (AUC, PSD, Ampl) compared with controls ( $10 \mathrm{mmol} / \mathrm{L}$ glucose); lactate significantly increased the frequency of the oscillations.

Supporting lactate $(20 \mathrm{mmol} / \mathrm{L})$ with glutamine $(2 \mathrm{mmol} / \mathrm{L})$, which is an important precursor for neurotransmitters, glutamate, and GABA (Waagepetersen et al., 1998; Hertz et al., 2014), did not rescue the amplitude of gamma oscillations (Figure 5).

These experiments show that a high concentration of either lactate or pyruvate can basically power gamma oscillations but alters their characteristics.

\section{GLYCOGEN STORES AND INHIBITION OF GLYCOGEN PHOSPHORYLASE}

We further explored whether glycogen breakdown is capable to fuel gamma oscillations. Glycogen stores have been described in astrocytes and, more recently, also in neurons (Choi et al., 2012; Dienel and Cruz, 2014; Saez et al., 2014). At first, we determined the time course of suppression of gamma oscillations during glucose deprivation in the presence of $95 \%$ oxygen fraction (Figures 6A-C). Activity was completely suppressed after cultures of the rat. (B) Corresponding power spectra of sample traces shown in (A) were calculated from $100 \mathrm{~s}$ taken at the end of each data segment. (C) Gamma oscillations were analyzed for various parameters, i.e., peak frequency (Freq), area under curve (AUC), full width at half maximum (FWHM), peak power spectral density (PSD), amplitude (Ampl), and variance of the amplitude (Var). Friedman repeated-measures ANOVA on ranks and Tukey post-hoc test. Statistical significance is marked by asterisks $(P<0.05)$.
$29 \pm 1 \mathrm{~min}(n=10)$ in recording solution with $0 \mathrm{mmol} / \mathrm{L}$ glucose (data not shown). This time course reflects utilization of various energy reserves such as glycogen for ATP generation in different pathways (Roberts, 2007; Dienel and Cruz, 2014) and, presumably, a considerable glycogen reserve in slice cultures (Cater et al., 2001).

We next pharmacologically blocked glycogen phosphorylase, which is a crucial enzyme for glycogen breakdown and thus serves in the initiation of glycogen metabolism (Dienel and Cruz, 2014). We applied two different inhibitors, i.e., DAB and CP-316819, at various concentrations (Gibbs et al., 2006; Dienel et al., 2007; Suh et al., 2007; Walls et al., 2008; Sickmann et al., 2009). Blockade of glycogen phosphorylase resulted in significantly faster suppression of gamma oscillations of $8 \pm 1 \mathrm{~min}(n>10)$ during glucose deprivation, indicating that glycogen breakdown can indeed support the maintenance of fast neuronal-network oscillations and thus higher brain functions in situations when glucose supply becomes limited (Wender et al., 2000; Abdelmalik et al., 2007).

We finally tested whether the turnover of glycogen was essential for sustainment of gamma oscillations. After gamma oscillations had been fully established, bath application of DAB $(100 \mu \mathrm{mol} / \mathrm{L})$ or CP-316819 $(20 \mu \mathrm{mol} / \mathrm{L})$ for $20 \mathrm{~min}$ left gamma oscillations widely intact (Figures 7A-C). We even partially observed increases in power and frequency of the oscillations.

These data show that glycogen is an important fuel reserve to sustain gamma oscillations for a transient period under pathological conditions such as hypoglycemia. However, the turnover of glycogen does not significantly contribute to sustainment of gamma oscillations when glucose is present. 


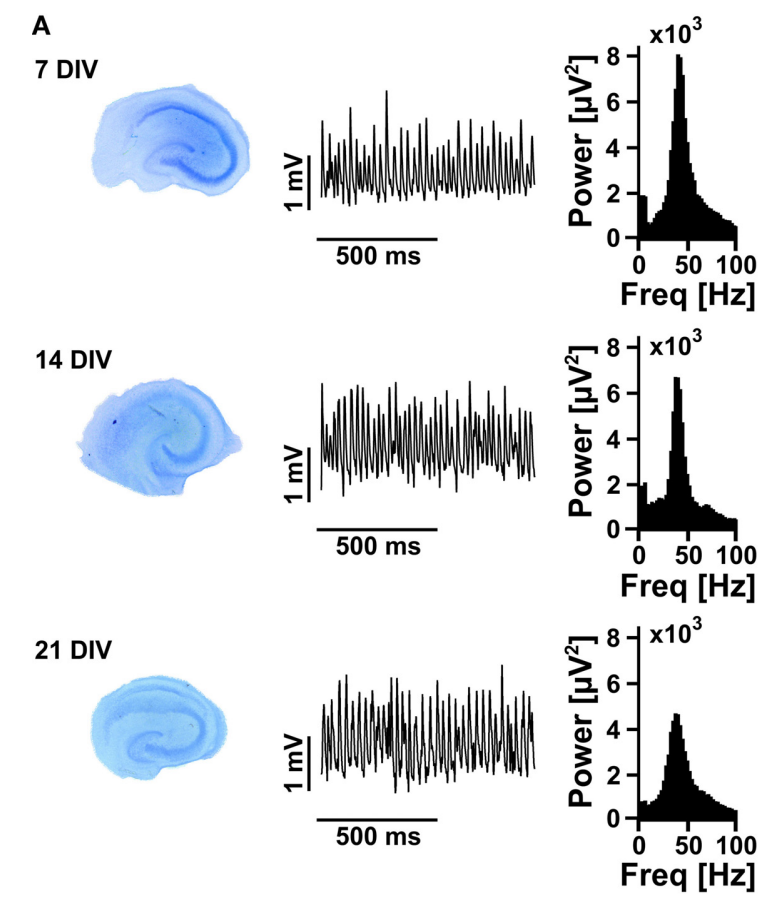

B
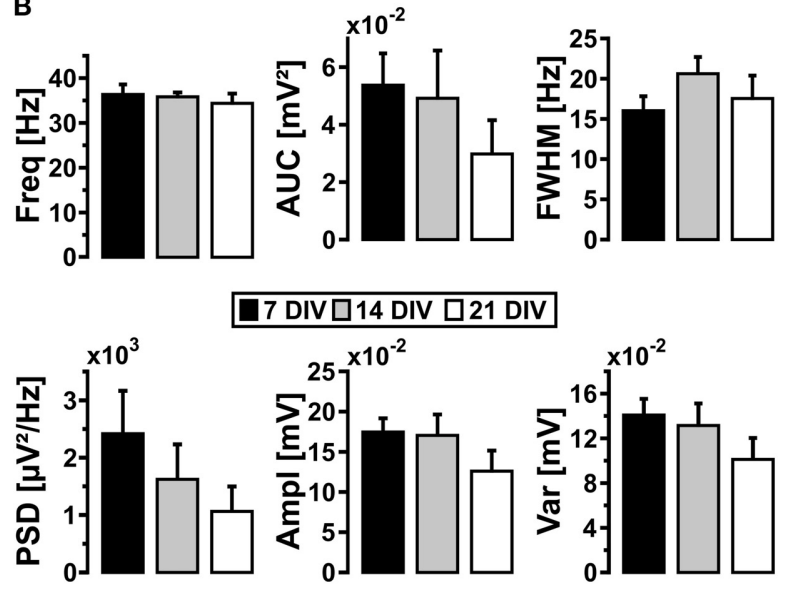

FIGURE 2 | Gamma oscillations and maturation of slice cultures. (A) Slice cultures were stained with toluidine blue after 7, 14, and 21 DIV (left). Gamma oscillations were induced by bath application of acetylcholine $(2 \mu \mathrm{mol} / \mathrm{L})$ and physostigmine $(400 \mathrm{nmol} / \mathrm{L})$ and local field potentials (LFP) were recorded in stratum pyramidale of the CA3 subfield (middle).

Corresponding power spectra of the sample traces were calculated from data segments of $100 \mathrm{~s}$ (right). (B) Gamma oscillations were analyzed for various parameters, i.e., peak frequency (Freq), area under curve (AUC), full width at half maximum (FWHM), peak power spectral density (PSD), amplitude (Ampl) and variance of the amplitude (Var) (7DIV, $n=12, N=4$; $14 \mathrm{DIV}, n=7, N=3 ; 21 \mathrm{DIV}, n=6, N=3)$. One-Way ANOVA and Kruskal-Wallis ANOVA on ranks.

\section{DISCUSSION}

\section{GAMMA OSCILLATIONS IN ORGANOTYPIC HIPPOCAMPAL SLICE CULTURES}

Gamma oscillations provide a temporal matrix for complex neuronal information processing during higher brain functions such as sensory perception, motor activity, and memory formation (Paulsen and Moser, 1998; Uhlhaas and Singer, 2010; Kann et al., 2014).

Persistent gamma oscillations can be reliably induced in acute slices and slice cultures of the hippocampus by bath application of cholinergic receptor agonists such as acetylcholine, which mimics cholinergic neuronal input from the septum in vivo (Bartos et al., 2007). Acetylcholine activates primarily muscarinic receptors in pyramidal cells and interneurons that interact via complex synaptic mechanisms (Bartos et al., 2007; Hájos and Paulsen, 2009). Cholinergic receptor activation finally leads to subthreshold membrane potential fluctuations in neurons and concomitant rhythmic network oscillations as detected by extracellular recordings of the LFP (Hájos et al., 1998; Fisahn et al., 2002; Kann et al., 2014). Notably, fast rhythmic GABAergic inhibition by parvalbumin-positive fast-spiking interneurons is crucial for the generation of gamma oscillations (Bartos et al., 2007; Gulyás et al., 2010; Oren et al., 2010). Cholinergically induced hippocampal gamma oscillations in vitro are most prominent in the CA3 subfield (Fisahn et al., 1998; Kann et al., 2011), which is similar to the pattern of gamma oscillations in vivo (Penttonen et al., 1998; Montgomery and Buzsáki, 2007). Gamma oscillations in vivo occur transiently on the $100 \mathrm{~ms}$ time scale upon sensory input or during specific cognitive tasks. In the human brain, for example, gamma oscillations can last in the range of minutes, dependent on the task (Lehmann et al., 2001; Lutz et al., 2004). This aspect is important for the validation of in vitro models, most of which feature persistent gamma oscillations (Bartos et al., 2007; Hájos and Paulsen, 2009).

Recent in vitro and in vivo studies demonstrated that gamma oscillations were associated with high energy expenditure (Niessing et al., 2005; Nishida et al., 2008; Kann et al., 2011; Huchzermeyer et al., 2013), which is most likely caused by increased rates of action potentials and synaptic interactions. In particular, the significant increase in excitatory and inhibitory postsynaptic potentials during gamma oscillations elicits strong ion fluxes across the neuronal membrane of pyramidal cells and inhibitory interneurons that finally need to be restored by ATPdependent ion pumps such as $\mathrm{Na}^{+} / \mathrm{K}^{+}$-ATPase (Kann et al., 2014).

Taken together, persistent gamma oscillations in organotypic hippocampal slice cultures are a useful model for a specific neuronal network activity state with high energy expenditure that naturally occurs in vivo.

\section{GLUCOSE, LACTATE AND PYRUVATE AS ENERGY SUBSTRATES}

Here we show that gamma oscillations reliably persist for more than $1 \mathrm{~h}$ in standard recording condition, i.e., in the presence of $10 \mathrm{mmol} / \mathrm{L}$ glucose and $95 \%$ oxygen fraction. Traditionally, brain slices are maintained in the presence of $10 \mathrm{mmol} / \mathrm{L}$ glucose for two main reasons: (i) improved recovery from the preparation procedure and (ii) heterogeneity in glucose and oxygen availability owing to the use of interface or submerged conditions, which also includes different application rates of recording solution (Li and Mcllwain, 1957; Kann and Kovács, 2007). Our optimized recording condition, i.e., the combination of slice cultures, Biopore ${ }^{\mathrm{TM}}$ membranes, and interface recording chamber, 


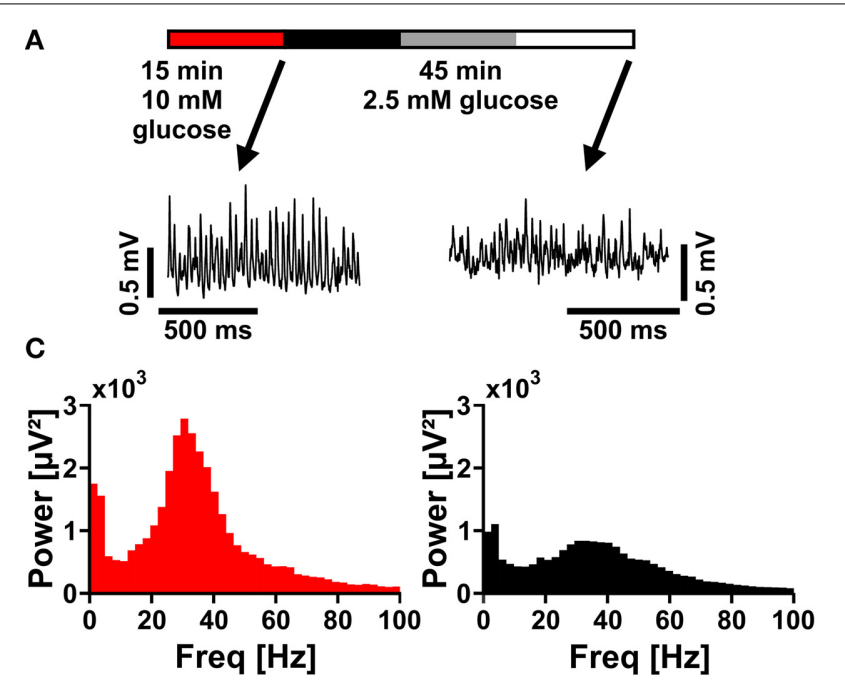

FIGURE 3 | Gamma oscillations at low glucose concentration. (A) Gamma oscillations were induced by bath application of acetylcholine $(2 \mu \mathrm{mol} / \mathrm{L})$ and physostigmine $(400 \mathrm{nmol} / \mathrm{L})$ in the presence of $10 \mathrm{mmol} / \mathrm{L}$ glucose (red). Then, glucose was lowered to $2.5 \mathrm{mmol} / \mathrm{L}$ in the recording solution and the properties of gamma oscillations were analyzed after $15 \mathrm{~min}$ (black), $30 \mathrm{~min}$ (gray), and $45 \mathrm{~min}$ (white). Local field potentials (LFP) were recorded in stratum pyramidale of the CA3 subfield (sample traces). (B) Corresponding power spectra of sample traces shown in (A) were calculated
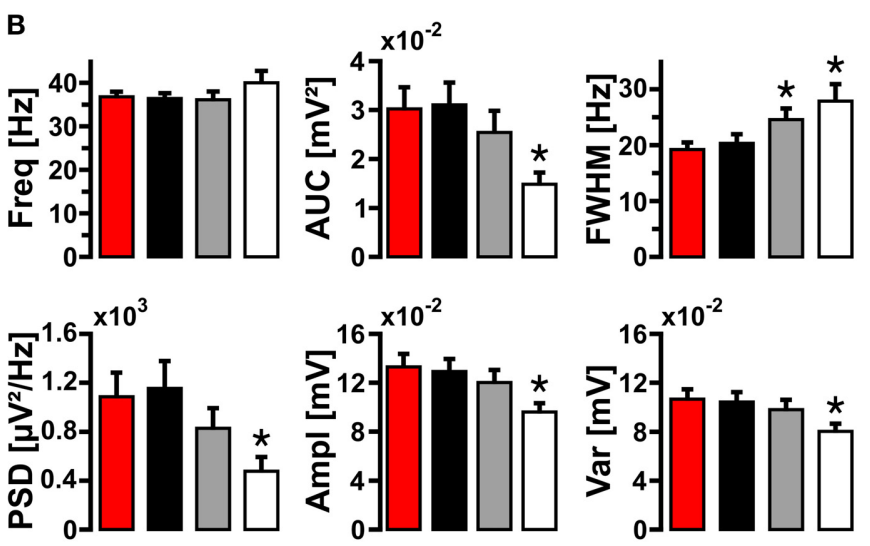

features superior supply of energy substrates and oxygen as well as fast drug application (Huchzermeyer et al., 2013).

However, gamma oscillations showed clear disturbances in the presence of $2.5 \mathrm{mmol} / \mathrm{L}$ glucose, even in the optimized recording condition. This finding differs from experiments in acute hippocampal slices, in which $2.5 \mathrm{mmol} / \mathrm{L}$ glucose were sufficient to sustain neuronal population responses as evoked by electrical stimulation (Schurr and Payne, 2007). Our experiments with energy substrates other than glucose showed that a high concentration $(20 \mathrm{mmol} / \mathrm{L})$ of lactate or pyruvate could indeed sustain gamma oscillations, but these oscillations were of significantly lower amplitude. This is also in contrast with other studies showing that lactate at $2 \mathrm{mmol} / \mathrm{L}$ or higher could maintain synaptic function in hippocampal tissue in vitro as well as, or better than, glucose (Schurr et al., 1988; Schurr and Payne, 2007; Ivanov et al., 2011) and/or proposing that lactate is the preferred energy substrate of neurons (Bouzier-Sore et al., 2003).

These different findings on the capability of low glucose or lactate to sustain neuronal activity are most likely due to (i) the respective activity state that is induced in the local neuronal network and/or (ii) intracellular acidification of neurons in the presence of lactate or pyruvate.

(i) In previous studies, electrical stimulation with either single (Schurr et al., 1988; Schurr and Payne, 2007) or repetitive pulses (at $10 \mathrm{~Hz}$, for 10 or $30 \mathrm{~s}$ ) (Ivanov et al., 2011) was used in acute hippocampal slices. Such electrical stimulation is quite robust and brief, and it enforces all neurons in the local network to generate action potentials. The final individual action potential frequency may considerably vary depending on neuronal subtypes and accommodation from $100 \mathrm{~s}$ taken at the end of each data segment. (C) Gamma oscillations were analyzed for various parameters, i.e., peak frequency (Freq), area under curve (AUC), full width at half maximum (FWHM), peak power spectral density (PSD), amplitude (Ampl), and variance of the amplitude (Var) ( $n=14$, $N=3)$. Note the decrease in amplitude at low glucose concentration. Repeated-measures ANOVA and Holm-Sidak post-hoc test or Friedman repeated-measures ANOVA on ranks and Tukey post-hoc test. Statistical significance is marked by asterisks $(P<0.05)$. characteristics (Kann et al., 2014). By contrast, hippocampal gamma oscillations are a specific, naturally occurring network activity state that is based on complex and precise synaptic interactions between excitatory pyramidal cells and inhibitory GABAergic interneurons (Whittington and Traub, 2003; Bartos et al., 2007; Hájos and Paulsen, 2009). During gamma oscillations, fast-spiking interneurons generate action potentials at $30-100 \mathrm{~Hz}$ while pyramidal cells spike at $1-3 \mathrm{~Hz}$ in vitro and in vivo (Csicsvari et al., 1999; Traub et al., 2000; Hájos et al., 2004; Gloveli et al., 2005). In particular, parvalbumin-positive fast-spiking interneurons are crucial for the generation of gamma oscillations and have very special characteristics such as formation of a basket cell network (Ribak et al., 1993; Traub et al., 2001), perisomatic control of pyramidal cells (Hájos et al., 2004; Gloveli et al., 2005), rapid action potential kinetics and high sodium entry ratio (Carter and Bean, 2009; Hu and Jonas, 2014). Moreover, parvalbumin-positive fast-spiking interneurons contain large numbers of mitochondria (Kageyama and Wong-Riley, 1982; Gulyás et al., 2006; Takács et al., 2014), which likely reflects the extraordinary high energy expenditure of this neuronal subtype during fast network oscillations (Kann et al., 2014). In agreement with these biophysical and biochemical characteristics, fast-spiking interneurons and gamma oscillations are exquisitely sensitive to metabolic stress (Huchzermeyer et al., 2008; Kann et al., 2011; Whittaker et al., 2011). The inability of $2 \mathrm{mmol} / \mathrm{L}$ glucose and $20 \mathrm{mmol} / \mathrm{L}$ lactate or pyruvate to (fully) sustain gamma oscillations might thus be related to properties of fast-spiking interneurons such as high energy expenditure, limitations in the uptake of substrates via glucose transporter GLUT-3 and 


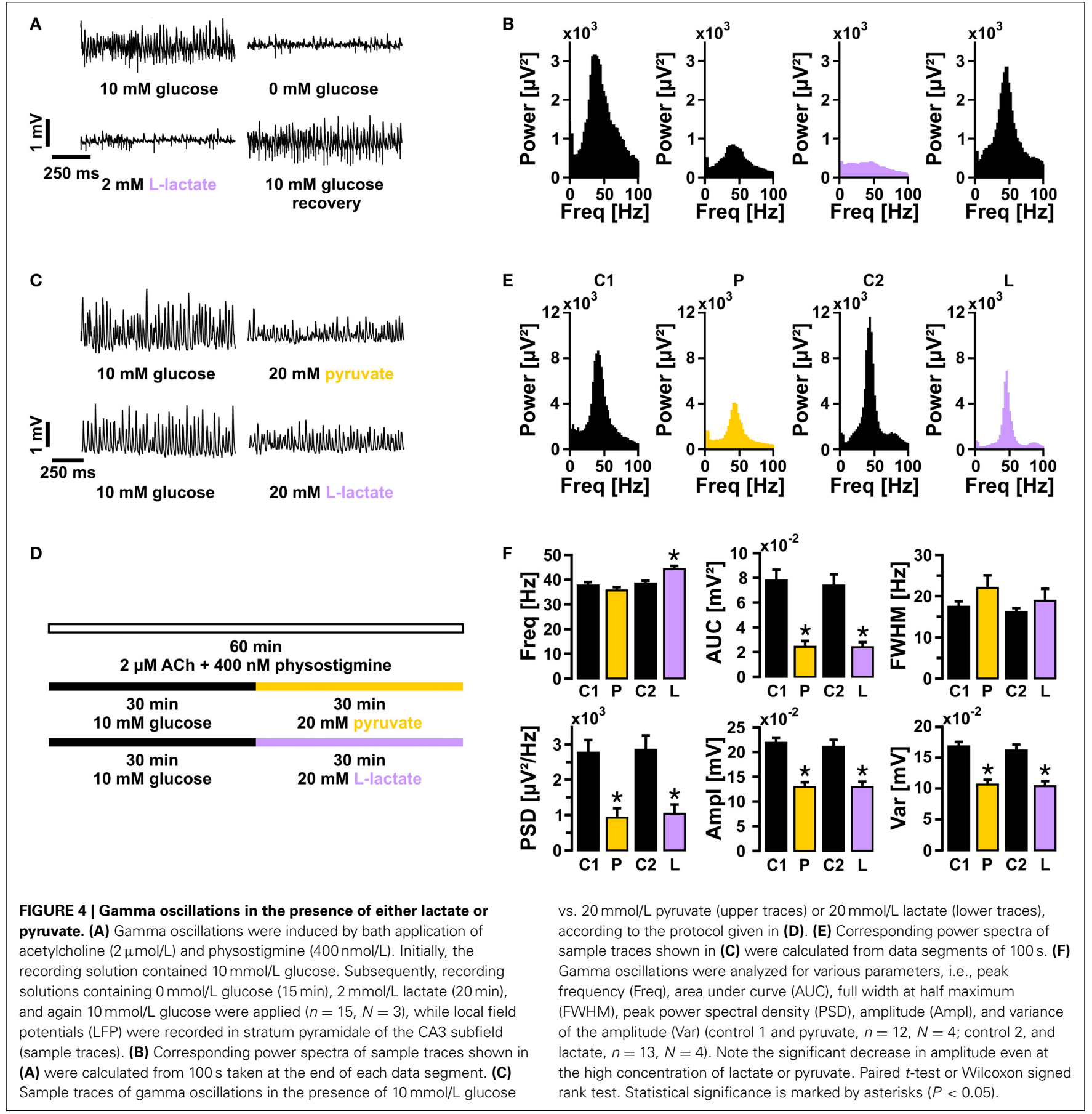

monocarboxylate transporter MCT-2 and/or limitations by rate-limiting enzymes in cytoplasmic and mitochondrial pathways related to energy and neurotransmitter metabolism (Waagepetersen et al., 1998; Bak et al., 2006; Simpson et al., 2007; Barros, 2013). However, the properties of transporters and enzymes in parvalbumin-positive fast-spiking interneurons are widely unknown (Kann et al., 2014).

(ii) A complementary or alternative explanation for the disturbances in gamma oscillations might be intracellular neuronal acidification in the presence of high concentration of lactate or pyruvate. Both substrates are taken up by neurons via MCT-2, which is a proton-linked monocarboxylate transporter (Roberts, 2007; Halestrap, 2013). Previous studies indeed showed decreases in the intracellular $\mathrm{pH}$ in the presence of $20 \mathrm{mmol} / \mathrm{L}$ lactate (Munsch and Pape, 1999; Ruusuvuori et al., 2010).

\section{GLYCOGEN STORES AND INHIBITION OF GLYCOGEN PHOSPHORYLASE}

Glycogen phosphorylase catalyzes the rate-limiting step in glycogenolysis in animals by releasing glucose-1-phosphate from 


\section{A}
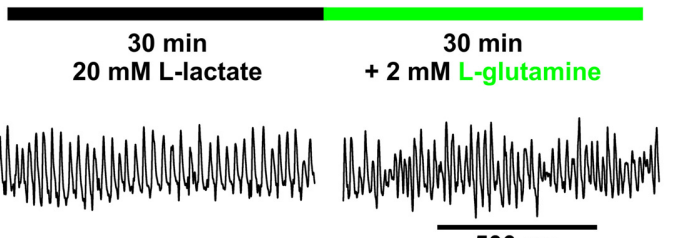

B

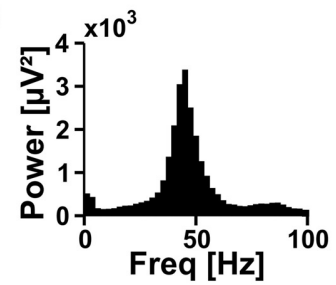

$500 \mathrm{~ms}$

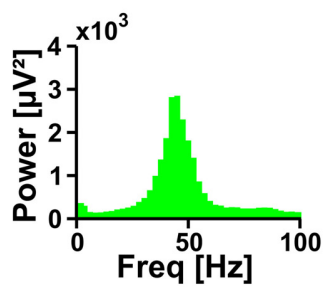

FIGURE 5 | Gamma oscillations in the presence of lactate and glutamine. (A) Gamma oscillations were induced by bath application of acetylcholine $(2 \mu \mathrm{mol} / \mathrm{L})$ and physostigmine $(400 \mathrm{nmol} / \mathrm{L})$ in the presence of $20 \mathrm{mmol} / \mathrm{L}$ lactate (black bar); after $30 \mathrm{~min}, 2 \mathrm{mmol} / \mathrm{L}$ glutamine was added (green bar). Local field potentials (LFP) were recorded in stratum pyramidale of the CA3 subfield (sample traces). (B) Corresponding power spectra of sample traces shown in (A) were calculated from
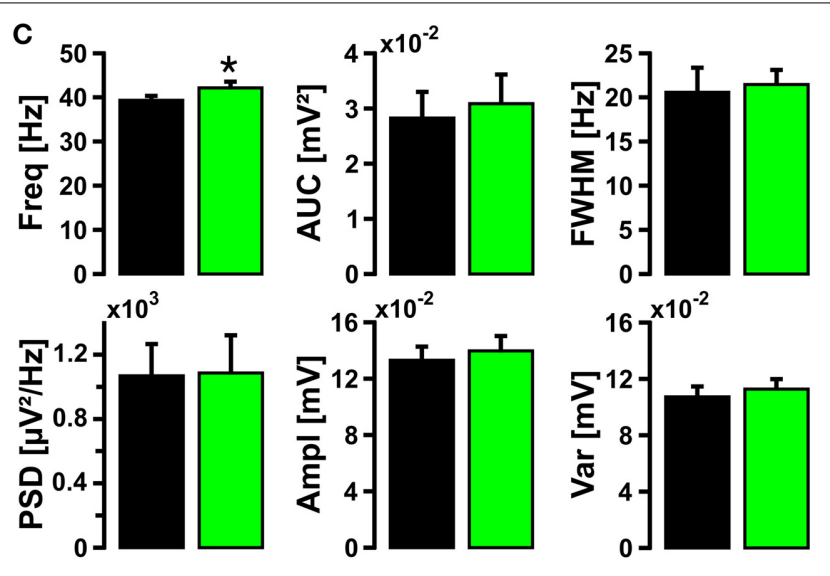

$100 \mathrm{~s}$ taken at the end of each data segment. (C) Gamma oscillations were analyzed for various parameters, i.e., peak frequency (Freq), area under curve (AUC), full width at half maximum (FWHM), peak power spectral density (PSD), amplitude (Ampl), and variance of the amplitude (Var) ( $n=19, N=4)$. Note that glutamine has only a minor effect on the frequency of gamma oscillations. Paired $t$-test. Statistical significance is marked by asterisks $(P<0.05)$.
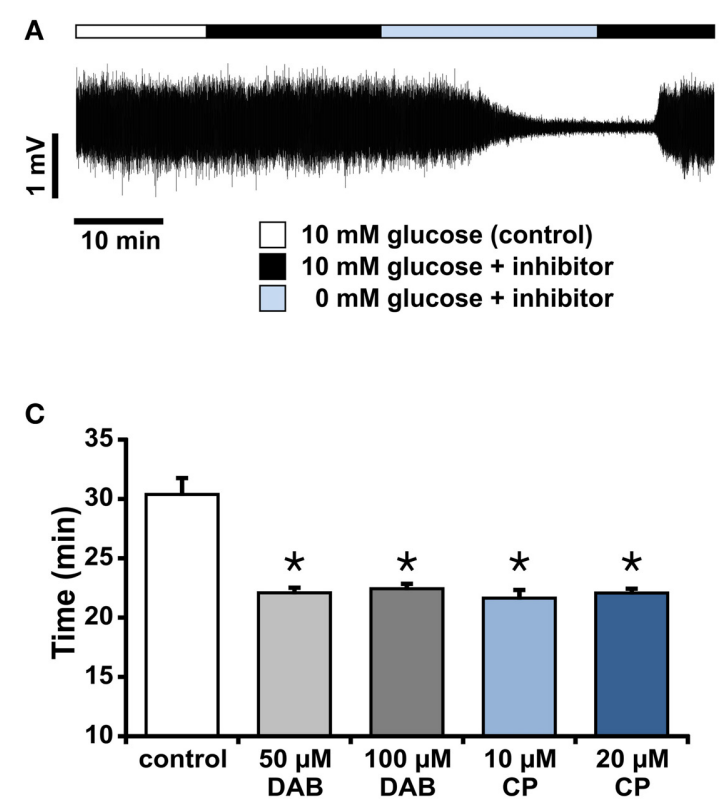

FIGURE 6 | Gamma oscillations and glycogen stores during glucose deprivation. (A) Gamma oscillations were induced by bath application of acetylcholine $(2 \mu \mathrm{mol} / \mathrm{L})$ and physostigmine $(400 \mathrm{nmol} / \mathrm{L})$ in the presence of $10 \mathrm{mmol} / \mathrm{L}$ glucose (white bar). Subsequently, inhibitors of glycogen phosphorylase, DAB $(50 \mu \mathrm{mol} / \mathrm{l}$ or $100 \mu \mathrm{mol} / \mathrm{L})$ or CP-316819 $(10 \mu \mathrm{mol} / \mathrm{L}$ or $20 \mu \mathrm{mol} / \mathrm{L}$ ) were applied, in the presence (black bar) or absence (light blue bar) of glucose. Note that the standard gas mixture (95\% O2 and 5\% CO2) was continuously present. Local field potentials (LFP) were recorded in stratum pyramidale of the CA3 subfield subfield (sample trace). (B) The peak power spectral density $\left(\mu \mathrm{V}^{2} / \mathrm{Hz}\right)$ for each recording trace is shown in black
B
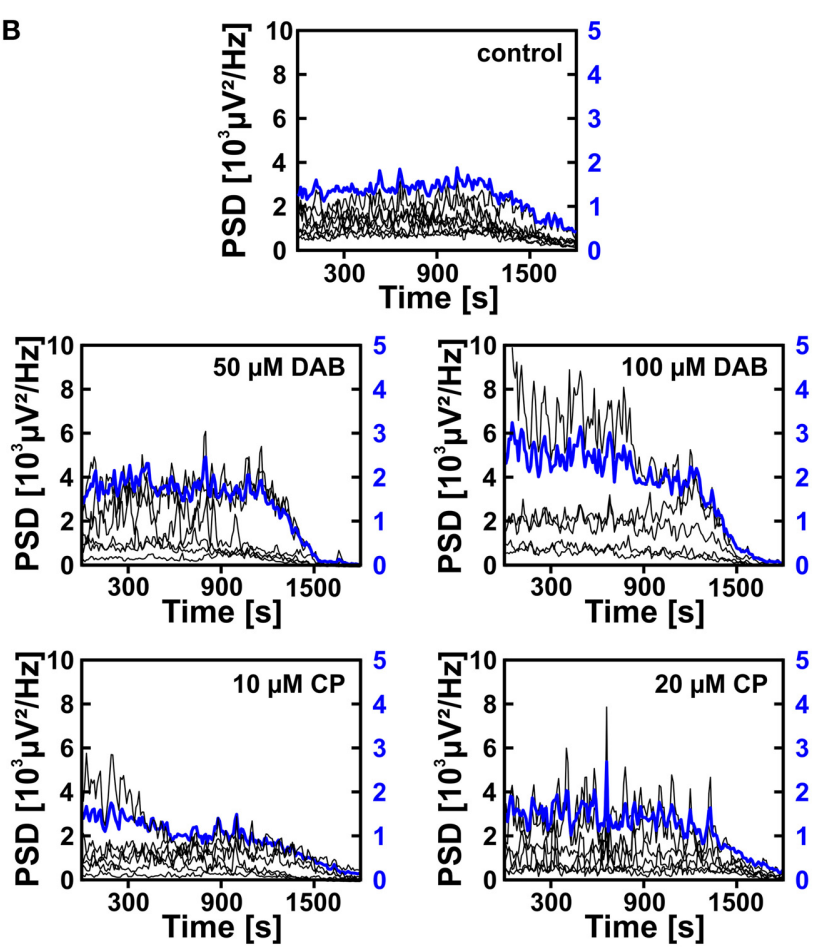

(scaling on left y-axis), the average of all recordings is shown in blue (scaling on right y-axis). Power spectra were calculated every $10 \mathrm{~s}$ and plotted over time. (C) The points in time are given for complete suppression of gamma oscillations, i.e., power reaching a threshold defined as the mean of the last $100 \mathrm{~s}$ plus 1 standard deviation, according to the protocol given in (A) (control, $n=10, N=3$; DAB, $n=6, N=3$, and $n=5, N=3$; CP-316819, $n=6, N=3$, and $n=5, N=2$ ). Note that inhibition of glycogen phosphorylase accelerates the decay of gamma oscillations during glucose deprivation. Kruskal Wallis ANOVA on ranks. Statistical significance vs. control is marked by asterisks $(P<0.05)$. 


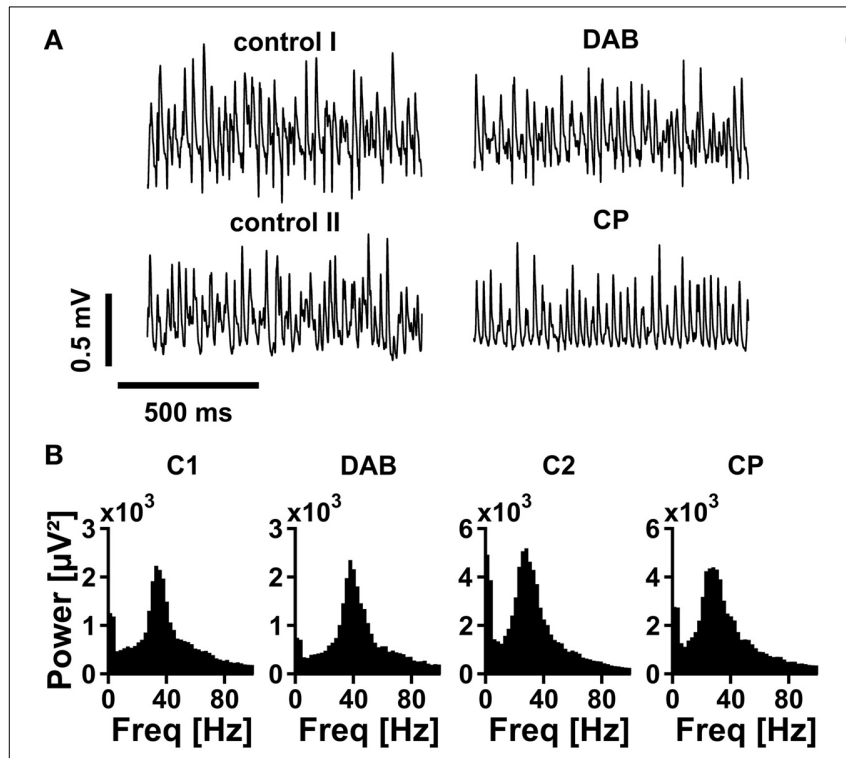

FIGURE 7 | Gamma oscillations and glycogen turnover. (A) Gamma oscillations were induced by bath application of acetylcholine $(2 \mu \mathrm{mol} / \mathrm{L})$ and physostigmine $(400 \mathrm{nmol} / \mathrm{L})$ in the presence of $10 \mathrm{mmol} / \mathrm{L}$ glucose. After $15 \mathrm{~min}$, inhibitors of glycogen phosphorylase, DAB $(50 \mu \mathrm{mol} / /$ or $100 \mu \mathrm{mol} / \mathrm{L})$ or CP-316819 $(10 \mu \mathrm{mol} / \mathrm{L}$ or $20 \mu \mathrm{mol} / \mathrm{L})$ were added to the recording solution Local field potentials (LFP) were recorded in stratum pyramidale of the CA3 subfield (sample traces). (B) Corresponding power spectra of sample traces shown in (A) were calculated from 100 s taken at the end of each data
C
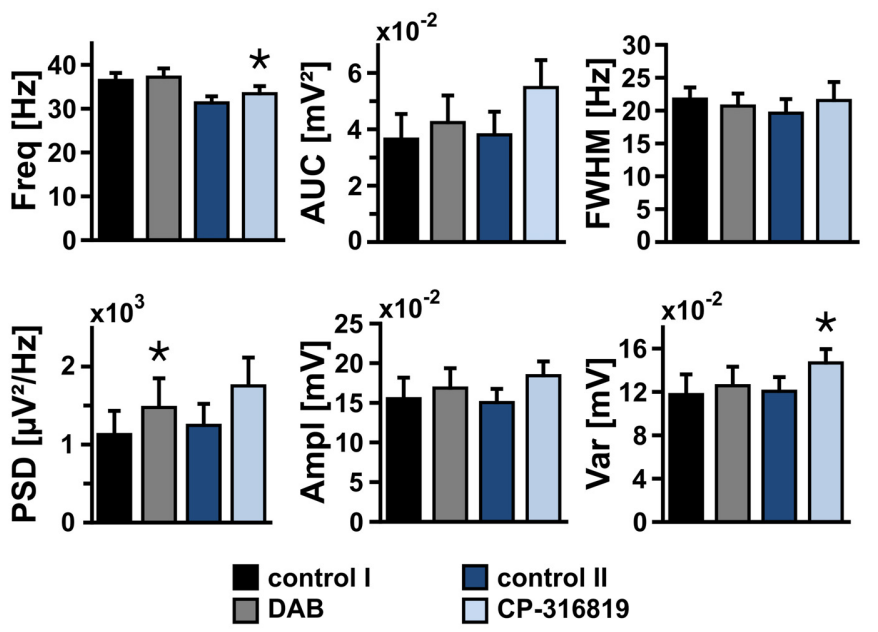

segment. (C) Gamma oscillations were analyzed for various parameters, i.e., peak frequency (Freq), area under curve (AUC), full width at half maximum (FWHM), peak power spectral density (PSD), amplitude (Ampl), and variance of the amplitude (Var) (control 1 and DAB, $n=13, N=4$; control 2 and CP-316819, $n=10, N=3)$. Note that inhibition of glycogen phosphorylase in the presence of $10 \mathrm{mmol} / \mathrm{L}$ glucose has only minor effects on gamma oscillations. Paired $t$-test or Wilcoxon signed rank test. Statistical significance is marked by asterisks $(P<0.05)$. the terminal alpha-1,4-glycosidic bond, finally supporting various intracellular metabolic pathways (Dienel and Cruz, 2014). It is widely accepted that glycogen is stored in astrocytes (Roberts, 2007; Dienel and Cruz, 2014). However, glycogen has been also found in localized compartments of neurons such as synaptic boutons and dendritic spines (Fiala et al., 2003), and there is recent evidence that neurons have an active glycogen metabolism (Saez et al., 2014). Several studies support two roles of glycogen as an energy substrate. The first role is to supply energy for regular neuronal activity (Swanson, 1992; Dienel et al., 2002; Kong et al., 2002; Gibbs et al., 2006; Choi et al., 2012; Duran et al., 2013). The second role is to provide glucose equivalents when supply with glucose or oxygen is limited, such as during hypoglycemia or ischemia/hypoxia (Wender et al., 2000; Choi et al., 2003; Saez et al., 2014). The glycogen metabolism might primarily occur in astrocytes that finally provide lactate to neurons (Dringen et al., 1993; Dienel and Cruz, 2014).

In our study, glycogen breakdown significantly delayed the decay of gamma oscillations in the absence of glucose for about $8 \mathrm{~min}$. This indicates (i) a considerable glycogen reserve in slice cultures that might be larger than in vivo (Cater et al., 2001; Kong et al., 2002), and (ii) quite effective mechanisms to mobilize and metabolize astrocytic and neuronal glycogen during gamma oscillations. However, inhibition of glycogen phosphorylase had only minor effects on gamma oscillations in the presence of glucose. Thus, our data suggest that glycogen serves as an important fuel reserve to sustain gamma oscillations and thus higher brain functions for a transient period under pathological conditions.

\section{REFERENCES}

Abdelmalik, P. A., Shannon, P., Yiu, A., Liang, P., Adamchik, Y., Weisspapir, M., et al. (2007). Hypoglycemic seizures during transient hypoglycemia exacerbate hippocampal dysfunction. Neurobiol. Dis. 26, 646-660. doi: 10.1016/j.nbd.2007.03.002

Attwell, D., Buchan, A. M., Charpak, S., Lauritzen, M., MacVicar, B. A., and Newman, E. A. (2010). Glial and neuronal control of brain blood flow. Nature 468, 232-243. doi: 10.1038/nature09613

Bahr, B. A., Kessler, M., Rivera, S., Vanderklish, P. W., Hall, R. A., Mutneja, M. S., et al. (1995). Stable maintenance of glutamate receptors and other synaptic components in long-term hippocampal slices. Hippocampus 5, 425-439. doi: 10.1002/hipo.450050505

Bak, L. K., Schousboe, A., and Waagepetersen, H. S. (2006). The glutamate/GABAglutamine cycle: aspects of transport, neurotransmitter homeostasis and ammonia transfer. J. Neurochem. 98, 641-653. doi: 10.1111/j.1471-4159.2006.03913.x

Barros, L. F. (2013). Metabolic signaling by lactate in the brain. Trends Neurosci. 36, 396-404. doi: 10.1016/j.tins.2013.04.002

Bartos, M., Vida, I., and Jonas, P. (2007). Synaptic mechanisms of synchronized gamma oscillations in inhibitory interneuron networks. Nat. Rev. Neurosci. 8, 45-56. doi: 10.1038/nrn2044

Bouzier-Sore, A.-K., Voisin, P., Canioni, P., Magistretti, P. J., and Pellerin, L. (2003). Lactate is a preferential oxidative energy substrate over glucose for neurons in culture. J. Cereb. Blood Flow Metab. 23, 1298-1306. doi: 10.1097/01.WCB.0000091761.61714

Carter, B. C., and Bean, B. P. (2009). Sodium entry during action potentials of mammalian neurons: incomplete inactivation and reduced metabolic efficiency in fast-spiking neurons. Neuron 64, 898-909. doi: 10.1016/j.neuron.2009.12.011

Cater, H. L., Benham, C. D., and Sundstrom, L. E. (2001). Neuroprotective role of monocarboxylate transport during glucose deprivation in slice cultures of rat hippocampus. J. Physiol. 531, 459-466. doi: 10.1111/j.1469-7793.2001.0459i.x

Cater, H. L., Chandratheva, A., Benham, C. D., Morrison, B. III., and Sundstrom, L. E. (2003). Lactate and glucose as energy substrates during, and after, oxygen deprivation in rat hippocampal acute and cultured slices. J. Neurochem. 87, 1381-1390. doi: 10.1046/j.1471-4159.2003.02100.x 
Chih, C.-P., and Roberts, Jr. E. L. (2003). Energy substrates for neurons during neural activity: a critical review of the astrocyte-neuron lactate shuttle hypothesis. J. Cereb. Blood Flow Metab. 23, 1263-1281. doi: 10.1097/01.WCB.0000081369. $51727.6 \mathrm{~F}$

Choi, H. B., Gordon, G. R. J., Zhou, N., Tai, C., Rungta, R. L., Martinez, J., et al. (2012). Metabolic communication between astrocytes and neurons via bicarbonate-responsive soluble adenylyl cyclase. Neuron 75, 1094-1104. doi: 10.1016/j.neuron.2012.08.032

Choi, I.-Y., Seaquist, E. R., and Gruetter, R. (2003). Effect of hypoglycemia on brain glycogen metabolism in vivo. J. Neurosci. Res. 72, 25-32. doi: 10.1002/jnr.10574

Csicsvari, J., Hirase, H., Czurkó, A., Mamiya, A., and Buzsáki, G. (1999). Oscillatory coupling of hippocampal pyramidal cells and interneurons in the behaving rat. J. Neurosci. 19, 274-287.

De Simoni, A., Griesinger, C. B., and Edwards, F. A. (2003). Development of rat CA1 neurones in acute versus organotypic slices: role of experience in synaptic morphology and activity. J. Physiol. 550, 135-147. doi: 10.1113/jphysiol.2003.039099

Dienel, G. A. (2012). Brain lactate metabolism: the discoveries and the controversies. J. Cereb. Blood Flow Metab. 32, 1107-1138. doi: 10.1038/jcbfm.2011.175

Dienel, G. A., Ball, K. K., and Cruz, N. F. (2007). A glycogen phosphorylase inhibitor selectively enhances local rates of glucose utilization in brain during sensory stimulation of conscious rats: implications for glycogen turnover J. Neurochem. 102, 466-478. doi: 10.1111/j.1471-4159.2007.04595.x

Dienel, G. A., and Cruz, N. F. (2014). Contributions of glycogen to astrocytic energetics during brain activation. Metab. Brain Dis. doi: 10.1007/s11011-0149493-8. [Epub ahead of print].

Dienel, G. A., Wang, R. Y., and Cruz, N. F. (2002). Generalized sensory stimulation of conscious rats increases labeling of oxidative pathways of glucose metabolism when the brain glucose-oxygen uptake ratio rises. J. Cereb. Blood Flow Metab. 22, 1490-1502. doi: 10.1097/00004647-200212000-00009

Dringen, R., Gebhardt, R., and Hamprecht, B. (1993). Glycogen in astrocytes: possible function as lactate supply for neighboring cells. Brain Res. 623, 208-214. doi: 10.1016/0006-8993(93)91429-V

Duran, J., Saez, I., Gruart, A., Guinovart, J. J., and Delgado-García, J. M. (2013). Impairment in long-term memory formation and learning-dependent synaptic plasticity in mice lacking glycogen synthase in the brain. J. Cereb. Blood Flow Metab. 33, 550-556. doi: 10.1038/jcbfm.2012.200

Erecińska, M., and Silver, I. A. (2001). Tissue oxygen tension and brain sensitivity to hypoxia. Respir. Physiol. 128, 263-276. doi: 10.1016/S0034-5687(01) 00306-1

Fiala, J. C., Kirov, S. A., Feinberg, M. D., Petrak, L. J., George, P., Goddard, C. A., et al. (2003). Timing of neuronal and glial ultrastructure disruption during brain slice preparation and recovery in vitro. J. Comp. Neurol. 465, 90-103. doi: 10.1002/cne.10825

Fisahn, A., Pike, F. G., Buhl, E. H., and Paulsen, O. (1998). Cholinergic induction of network oscillations at $40 \mathrm{~Hz}$ in the hippocampus in vitro. Nature 394, 186-189. doi: 10.1038/28179

Fisahn, A., Yamada, M., Duttaroy, A., Gan, J.-W., Deng, C.-X., McBain, C. J., et al. (2002). Muscarinic induction of hippocampal gamma oscillations requires coupling of the M1 receptor to two mixed cation currents. Neuron 33, 615-624. doi: 10.1016/S0896-6273(02)00587-1

Galeffi, F., Foster, K. A., Sadgrove, M. P., Beaver, C. J., and Turner, D. A. (2007). Lactate uptake contributes to the $\mathrm{NAD}(\mathrm{P}) \mathrm{H}$ biphasic response and tissue oxygen response during synaptic stimulation in area CA1 of rat hippocampal slices. J. Neurochem. 103, 2449-2461. doi: 10.1111/j.1471-4159.2007.04939.x

Gandhi, G. K., Cruz, N. F., Ball, K. K., and Dienel, G. A. (2009). Astrocytes are poised for lactate trafficking and release from activated brain and for supply of glucose to neurons. J. Neurochem. 111, 522-536. doi: 10.1111/j.14714159.2009.06333.x

Gibbs, M. E., Anderson, D. G., and Hertz, L. (2006). Inhibition of glycogenolysis in astrocytes interrupts memory consolidation in young chickens. Glia 54, 214-222. doi: 10.1002/glia.20377

Gloveli, T., Dugladze, T., Saha, S., Monyer, H., Heinemann, U., Traub, R. D., et al. (2005). Differential involvement of oriens/pyramidale interneurones in hippocampal network oscillations in vitro. J. Physiol. 562, 131-147. doi: 10.1113/jphysiol.2004.073007

Gulyás, A. I., Buzsáki, G., Freund, T. F., and Hirase, H. (2006). Populations of hippocampal inhibitory neurons express different levels of cytochrome c. Eur. J. Neurosci. 23, 2581-2594. doi: 10.1111/j.1460-9568.2006.04814.x
Gulyás, A. I., Szabó, G. G., Ulbert, I., Holderith, N., Monyer, H., Erdélyi, F., et al. (2010). Parvalbumin-containing fast-spiking basket cells generate the field potential oscillations induced by cholinergic receptor activation in the hippocampus. J. Neurosci. 30, 15134-15145. doi: 10.1523/JNEUROSCI.410410.2010

Hájos, N., Pálhalmi, J., Mann, E. O., Németh, B., Paulsen, O., and Freund, T. F. (2004). Spike timing of distinct types of GABAergic interneuron during hippocampal gamma oscillations in vitro. J. Neurosci. 24, 9127-9137. doi: 10.1523/JNEUROSCI.2113-04.2004

Hájos, N., Papp, E. C., Acsády, L., Levey, A. I., and Freund, T. F. (1998). Distinct interneuron types express $\mathrm{m} 2$ muscarinic receptor immunoreactivity on their dendrites or axon terminals in the hippocampus. Neuroscience 82, 355-376. doi: 10.1016/S0306-4522(97)00300-X

Hájos, N., and Paulsen, O. (2009). Network mechanisms of gamma oscillations in the CA3 region of the hippocampus. Neural Netw. 22, 1113-1119. doi: 10.1016/j.neunet.2009.07.024

Halestrap, A. P. (2013). The SLC16 gene family - structure, role and regulation in health and disease. Mol. Aspects Med. 34, 337-349. doi: 10.1016/j.mam.2012.05.003

Hall, C. N., Klein-Flügge, M. C., Howarth, C., and Attwell, D. (2012). Oxidative phosphorylation, not glycolysis, powers presynaptic and postsynaptic mechanisms underlying brain information processing. J. Neurosci. 32, 8940-8951. doi: 10.1523/JNEUROSCI.0026-12.2012

Hertz, L., Gibbs, M. E., and Dienel, G. A. (2014). Fluxes of lactate into, from, and among gap junction-coupled astrocytes and their interaction with noradrenaline. Front. Neurosci. 8:261. doi: 10.3389/fnins.2014.00261

$\mathrm{Hu}, \mathrm{H}$. , and Jonas, P. (2014). A supercritical density of $\mathrm{Na}^{+}$channels ensures fast signaling in GABAergic interneuron axons. Nat. Neurosci. 17, 686-693. doi: 10.1038/nn.3678

Huchzermeyer, C., Albus, K., Gabriel, H.-J., Otáhal, J., Taubenberger, N., Heinemann, U., et al. (2008). Gamma oscillations and spontaneous network activity in the hippocampus are highly sensitive to decreases in $\mathrm{pO}_{2}$ and concomitant changes in mitochondrial redox state. J. Neurosci. 28, 1153-1162. doi: 10.1523/JNEUROSCI.4105-07.2008

Huchzermeyer, C., Berndt, N., Holzhütter, H.-G., and Kann, O. (2013). Oxygen consumption rates during three different neuronal activity states in the hippocampal CA3 network. J. Cereb. Blood Flow Metab. 33, 263-271. doi: 10.1038/jcbfm.2012.165

Ide, K., Schmalbruch, I. K., Quistorff, B., Horn, A., and Secher, N. H. (2000). Lactate, glucose and $\mathrm{O}_{2}$ uptake in human brain during recovery from maximal exercise. J. Physiol. 522, 159-164. doi: 10.1111/j.1469-7793.2000.t01-200159.xm

Ivanov, A. I., Malkov, A. E., Waseem, T., Mukhtarov, M., Buldakova, S., Gubkina, O., et al. (2014). Glycolysis and oxidative phosphorylation in neurons and astrocytes during network activity in hippocampal slices. J. Cereb. Blood Flow Metab. 34, 397-407. doi: 10.1038/jcbfm.2013.222

Ivanov, A., Mukhtarov, M., Bregestovski, P., and Zilberter, Y. (2011). Lactate effectively covers energy demands during neuronal network activity in neonatal hippocampal slices. Front. Neuroenergetics 3:2. doi: 10.3389/fnene.2011.00002

Kageyama, G. H., and Wong-Riley, M. T. T. (1982). Histochemical localization of cytochrome oxidase in the hippocampus: correlation with specific neuronal types and afferent pathways. Neuroscience 7, 2337-2361. doi: 10.1016/03064522(82)90199-3

Kann, O. (2012). The energy demand of fast neuronal network oscillations: insights from brain slice preparations. Front. Pharmacol. 2:90. doi: 10.3389/fphar.2011.00090

Kann, O., Huchzermeyer, C., Kovács, R., Wirtz, S., and Schuelke, M. (2011) Gamma oscillations in the hippocampus require high complex I gene expression and strong functional performance of mitochondria. Brain 134, 345-358. doi: 10.1093/brain/awq333

Kann, O., and Kovács, R. (2007). Mitochondria and neuronal activity. Am. J. Physiol. Cell Physiol. 292, C641-C657. doi: 10.1152/ajpcell.00222.2006

Kann, O., Kovács, R., and Heinemann, U. (2003a). Metabotropic receptormediated $\mathrm{Ca}^{2+}$ signaling elevates mitochondrial $\mathrm{Ca}^{2+}$ and stimulates oxidative metabolism in hippocampal slice cultures. J. Neurophysiol. 90, 613-621. doi: 10.1152/jn.00042.2003

Kann, O., Papageorgiou, I. E., and Draguhn, A. (2014). Highly energized inhibitory interneurons are a central element for information processing in cortical networks. J. Cereb. Blood Flow Metab. 34, 1270-1282. doi: 10.1038/jcbfm.2014.104 
Kann, O., Schuchmann, S., Buchheim, K., and Heinemann, U. (2003b). Coupling of neuronal activity and mitochondrial metabolism as revealed by $\mathrm{NAD}(\mathrm{P}) \mathrm{H}$ fluorescence signals in organotypic hippocampal slice cultures of the rat. Neuroscience 119, 87-100. doi: 10.1016/S0306-4522(03) 00026-5

Kong, J., Shepel, P. N., Holden, C. P., Mackiewicz, M., Pack, A. I., and Geiger, J. D. (2002). Brain glycogen decreases with increased periods of wakefulness: implications for homeostatic drive to sleep. J. Neurosci. 22, 5581-5587.

Lehmann, D., Faber, P. L., Achermann, P., Jeanmonod, D., Gianotti, L. R. R., and Pizzagalli, D. (2001). Brain sources of EEG gamma frequency during volitionally meditation-induced, altered states of consciousness, and experience of the self. Psychiatry Res. 108, 111-121. doi: 10.1016/S0925-4927(01) 00116-0

Li, C.-L., and McIlwain, H. (1957). Maintenance of resting membrane potentials in slices of mammalian cerebral cortex and other tissues in vitro. J. Physiol. 139, 178-190.

Lutz, A., Greischar, L. L., Rawlings, N. B., Ricard, M., and Davidson, R. J. (2004). Long-term meditators self-induce high-amplitude gamma synchrony during mental practice. Proc. Natl. Acad. Sci. U.S.A. 101, 16369-16373. doi: 10.1073/pnas.0407401101

Montgomery, S. M., and Buzsáki, G. (2007). Gamma oscillations dynamically couple hippocampal CA3 and CA1 regions during memory task performance. Proc. Natl. Acad. Sci. U.S.A. 104, 14495-14500. doi: 10.1073/pnas.0701 826104

Munsch, T., and Pape, H.-C. (1999). Modulation of the hyperpolarization-activated cation current of rat thalamic relay neurones by intracellular pH. J. Physiol. 519, 493-504. doi: 10.1111/j.1469-7793.1999.0493m.x

Niessing, J., Ebisch, B., Schmidt, K. E., Niessing, M., Singer, W., and Galuske, R. A. W. (2005). Hemodynamic signals correlate tightly with synchronized gamma oscillations. Science 309, 948-951. doi: 10.1126/science.1110948

Nishida, M., Juhász, C., Sood, S., Chugani, H. T., and Asano, E. (2008). Cortical glucose metabolism positively correlates with gamma-oscillations in nonlesional focal epilepsy. Neuroimage 42, 1275-1284. doi: 10.1016/j.neuroimage. 2008.06.027

Oren, I., Hájos, N., and Paulsen, O. (2010). Identification of the current generator underlying cholinergically induced gamma frequency field potential oscillations in the hippocampal CA3 region. J. Physiol. 588, 785-797. doi: 10.1113/jphysiol.2009.180851

Overgaard, M., Rasmussen, P., Bohm, A. M., Seifert, T., Brassard, P., Zaar, M., et al. (2012). Hypoxia and exercise provoke both lactate release and lactate oxidation by the human brain. FASEB J. 26, 3012-3020. doi: 10.1096/fj.11191999

Paulsen, O., and Moser, E. I. (1998). A model of hippocampal memory encoding and retrieval: GABAergic control of synaptic plasticity. Trends Neurosci. 21, 273-278. doi: 10.1016/S0166-2236(97)01205-8

Pellerin, L., and Magistretti, P. J. (2012). Sweet sixteen for ANLS. J. Cereb. Blood Flow Metab. 32, 1152-1166. doi: 10.1038/jcbfm.2011.149

Penttonen, M., Kamondi, A., Acsády, L., and Buzsáki, G. (1998). Gamma frequency oscillation in the hippocampus of the rat: intracellular analysis in vivo. Eur. J. Neurosci. 10, 718-728. doi: 10.1046/j.1460-9568.1998.00096.x

Ribak, C. E., Seress, L., and Leranth, C. (1993). Electron microscopic immunocytochemical study of the distribution of parvalbumin-containing neurons and axon terminals in the primate dentate gyrus and Ammon's horn. J. Comp. Neurol. 327, 298-321. doi: 10.1002/cne.903270211

Roberts, E. L. Jr. (2007). “The support of energy metabolism in the central nervous system with substrates other than glucose," in Handbook of Neurochemistry and Molecular Neurobiology, eds A. Lajtha, G. Gido, and G. A. Dienel (Berlin; Heidelberg: Springer-Verlag), 137-179. doi: 10.1007/978-0-387-30 411-3_7

Rolfe, D. F. S., and Brown, G. C. (1997). Cellular energy utilization and molecular origin of standard metabolic rate in mammals. Physiol. Rev. 77, 731-758.

Ruusuvuori, E., Kirilkin, I., Pandya, N., and Kaila, K. (2010). Spontaneous network events driven by depolarizing GABA action in neonatal hippocampal slices are not attributable to deficient mitochondrial energy metabolism. J. Neurosci. 30, 15638-15642. doi: 10.1523/JNEUROSCI.3355-10.2010

Saez, I., Duran, J., Sinadinos, C., Beltran, A., Yanes, O., Tevy, M. F., et al. (2014). Neurons have an active glycogen metabolism that contributes to tolerance to hypoxia. J. Cereb. Blood Flow Metab. 34, 945-955. doi: 10.1038/jcbfm. 2014.33
Schurr, A., and Gozal, E. (2012). Aerobic production and utilization of lactate satisfy increased energy demands upon neuronal activation in hippocampal slices and provide neuroprotection against oxidative stress. Front. Pharmacol. 2:96. doi: 10.3389/fphar.2011.00096

Schurr, A., Miller, J. J., Payne, R. S., and Rigor, B. M. (1999). An increase in lactate output by brain tissue serves to meet the energy needs of glutamate-activated neurons. J. Neurosci. 19, 34-39.

Schurr, A., and Payne, R. S. (2007). Lactate, not pyruvate, is neuronal aerobic glycolysis end product: an in vitro electrophysiological study. Neuroscience 147, 613-619. doi: 10.1016/j.neuroscience.2007.05.002

Schurr, A., West, C. A., and Rigor, B. M. (1988). Lactate-supported synaptic function in the rat hippocampal slice preparation. Science 240, 1326-1328. doi: $10.1126 /$ science.3375817

Shulman, R. G., Hyder, F., and Rothman, D. L. (2001). Lactate efflux and the neuroenergetic basis of brain function. NMR Biomed. 14, 389-396. doi: 10.1002/nbm.741

Sickmann, H. M., Walls, A. B., Schousboe, A., Bouman, S. D., and Waagepetersen, H. S. (2009). Functional significance of brain glycogen in sustaining glutamatergic neurotransmission. J. Neurochem. 109 Suppl. 1, 80-86. doi: 10.1111/j.14714159.2009.05915.x

Simpson, I. A., Carruthers, A., and Vannucci, S. J. (2007). Supply and demand in cerebral energy metabolism: the role of nutrient transporters. J. Cereb. Blood Flow Metab. 27, 1766-1791. doi: 10.1038/sj.jcbfm.9600521

Stittsworth, J. D. Jr., and Lanthorn, T. H. (1993). Lactate mimics only some effects of D-glucose on epileptic depolarization and long-term synaptic failure. Brain Res. 630, 21-27. doi: 10.1016/0006-8993(93)90637-3

Suh, S. W., Bergher, J. P., Anderson, C. M., Treadway, J. L., Fosgerau, K., and Swanson, R. A. (2007). Astrocyte glycogen sustains neuronal activity during hypoglycemia: studies with the glycogen phosphorylase inhibitor CP316,819 ([R-R $\left.R^{*}, S^{*}\right]$-5-chloro- $N$-[2-hydroxy-3-(methoxymethylamino)-3-oxo1-(phenylmethyl)pro pyl]-1H-indole-2-carboxamide). J. Pharmacol. Exp. Ther. 321, 45-50. doi: 10.1124/jpet.106.115550

Suzuki, A., Stern, S. A., Bozdagi, O., Huntley, G. W., Walker, R. H., Magistretti, P. J., et al. (2011). Astrocyte-neuron lactate transport is required for long-term memory formation. Cell 144, 810-823. doi: 10.1016/j.cell.2011. 02.018

Swanson, R. A. (1992). Physiologic coupling of glial glycogen metabolism to neuronal activity in brain. Can. J. Physiol. Pharmacol. 70 Suppl., S138-S144. doi: 10.1139/y92-255

Takács, V. T., Szönyi, A., Freund, T. F., Nyiri, G., and Gulyás, A. I. (2014). Quantitative ultrastructural analysis of basket and axo-axonic cell terminals in the mouse hippocampus. Brain Struct. Funct. doi: 10.1007/s00429-0130692-6. [Epub ahead of print].

Traub, R. D., Bibbig, A., Fisahn, A., LeBeau, F. E. N., Whittington, M. A., and Buhl, E. H. (2000). A model of gamma-frequency network oscillations induced in the rat CA3 region by carbachol in vitro. Eur. J. Neurosci. 12, 4093-4106. doi: 10.1046/j.1460-9568.2000.00300.x

Traub, R. D., Kopell, N., Bibbig, A., Buhl, E. H., LeBeau, F. E. N., and Whittington, M. A. (2001). Gap junctions between interneuron dendrites can enhance synchrony of gamma oscillations in distributed networks. J. Neurosci. 21, 9478-9486.

Uhlhaas, P. J., and Singer, W. (2010). Abnormal neural oscillations and synchrony in schizophrenia. Nat. Rev. Neurosci. 11, 100-113. doi: 10.1038/nrn2774

van Vugt, M. K., Schulze-Bonhage, A., Litt, B., Brandt, A., and Kahana, M. J. (2010). Hippocampal gamma oscillations increase with memory load. J. Neurosci. 30, 2694-2699. doi: 10.1523/JNEUROSCI.0567-09.2010

Waagepetersen, H. S., Bakken, I. J., Larsson, O. M., Sonnewald, U., and Schousboe, A. (1998). Metabolism of lactate in cultured GABAergic neurons studied by ${ }^{13} \mathrm{C}$ nuclear magnetic resonance spectroscopy. J. Cereb. Blood Flow Metab. 18, 109-117. doi: 10.1097/00004647-199801000-00011

Walls, A. B., Sickmann, H. M., Brown, A., Bouman, S. D., Ransom, B., Schousboe, A., et al. (2008). Characterization of 1,4-dideoxy-1,4-imino-d-arabinitol (DAB) as an inhibitor of brain glycogen shunt activity. J. Neurochem. 105, 1462-1470. doi: 10.1111/j.1471-4159.2008.05250.x

Wender, R., Brown, A. M., Fern, R., Swanson, R. A., Farrell, K., and Ransom, B. R. (2000). Astrocytic glycogen influences axon function and survival during glucose deprivation in central white matter. J. Neurosci. 20, 6804-6810.

Whittaker, R. G., Turnbull, D. M., Whittington, M. A., and Cunningham, M. O. (2011). Impaired mitochondrial function abolishes gamma oscillations in the 
hippocampus through an effect on fast-spiking interneurons. Brain 134, e180. doi: 10.1093/brain/awr018

Whittington, M. A., and Traub, R. D. (2003). Interneuron diversity series: inhibitory interneurons and network oscillations in vitro. Trends Neurosci. 26, 676-682. doi: 10.1016/j.tins.2003.09.016

Conflict of Interest Statement: The authors declare that the research was conducted in the absence of any commercial or financial relationships that could be construed as a potential conflict of interest.

Received: 14 October 2014; paper pending published: 01 November 2014; accepted: 18 November 2014; published online: 05 December 2014.
Citation: Galow LV, Schneider J, Lewen A, Ta T-T, Papageorgiou IE and Kann O (2014) Energy substrates that fuel fast neuronal network oscillations. Front. Neurosci. 8:398. doi: 10.3389/fnins.2014.00398

This article was submitted to Neuroenergetics, Nutrition and Brain Health, a section of the journal Frontiers in Neuroscience.

Copyright (C) 2014 Galow, Schneider, Lewen, Ta, Papageorgiou and Kann. This is an open-access article distributed under the terms of the Creative Commons Attribution License (CC BY). The use, distribution or reproduction in other forums is permitted, provided the original author(s) or licensor are credited and that the original publication in this journal is cited, in accordance with accepted academic practice. No use, distribution or reproduction is permitted which does not comply with these terms. 